INPLASY

PROTOCOL

To cite: Liu et al. Treatment of vitiligo with fire needle $A$ protocol for systematic review and meta analysis. Inplasy protocol 2020120012. doi: 10.37766/inplasy2020.12.0012

Received: 02 December 2020

Published: 02 December 2020

Corresponding author:

Liu Mingqiang

1099590012@qq.com

Author Affiliation: Jiangxi University of traditional Chinese medicine; Affiliated Hospital of Jiangxi University of traditional Chinese Medicine

Support: Affiliated Hospital of Jiangxi.

Review Stage at time of this submission: Preliminary searches.

Conflicts of interest: None.

\section{Treatment of vitiligo with fire needle A protocol for systematic review and meta analysis}

Liu, M; Qiu, G².

Review question / Objective: 2.1 Types of study All RCTs of fire needle therapy for vitiligo will be included without language restriction. Non-RCTs, observational studies, crossover studies, uncontrolled trials, animal trials, and reviews will be excluded. 2.1.2 Types of participants Inclusion criteria for study populations will be all patients with vitiligo. No restrictions will be applied in terms of gender, age, race, condition duration, or intensity. The language is limited to Chinese and English. 2.1.3 Types of interventions 2.1.3.1 Experimental interventions The treatment group will receive fire needle therapy and can be combined with external application of drugs and narrow-band violet External line, 308 excimer light, traditional Chinese medicine and other methods, without any restrictions on needle material, shape, or treatment process. 2.1.3.2 Control interventions The control group will receive an internationally recognized therapy such as external application of drugs and narrow-band violet External line, 308 excimer light, Placebo, no treatment, but not use fire needle treatment; 2.1.4 Types of outcome measures 2.1.4.1 Primary outcomes Complex area of white spot and Percentage of Clinical Effectiveness will be accepted as the primary outcomes. 2.1.4.2 Additional outcomes The safety assessment will be considered a secondary outcome

INPLASY registration number: This protocol was registered with the International Platform of Registered Systematic Review and Meta-Analysis Protocols (INPLASY) on 02 December 2020 and was last updated on 02 December 2020 (registration number INPLASY2020120012).

\section{INTRODUCTION}

Review question / Objective: 2.1 Types of study All RCTs of fire needle therapy for vitiligo will be included without language restriction. Non-RCTs, observational studies, cross-over studies, uncontrolled trials, animal trials, and reviews will be 
excluded. 2.1.2 Types of participants Inclusion criteria for study populations will be all patients with vitiligo. No restrictions will be applied in terms of gender, age, race, condition duration, or intensity. The language is limited to Chinese and English. 2.1.3 Types of interventions 2.1.3.1 Experimental interventions The treatment group will receive fire needle therapy and can be combined with external application of drugs and narrow-band violet External line, 308 excimer light, traditional Chinese medicine and other methods, without any restrictions on needle material, shape, or treatment process. 2.1.3.2 Control interventions The control group will receive an internationally recognized therapy such as external application of drugs and narrow-band violet External line, 308 excimer light, Placebo, no treatment, but not use fire needle treatment; 2.1.4 Types of outcome measures 2.1.4.1 Primary outcomes Complex area of white spot and Percentage of Clinical Effectiveness will be accepted as the primary outcomes. 2.1.4.2 Additional outcomes The safety assessment will be considered a secondary outcome

Condition being studied: Background:we aim to study the treatment of vitiligo with fire needle. Methods:we will search PubMed, Embase, the Cochrane Library, the China National Knowledge Infrastructure, Chinese Science and Technology Periodical Database, Wanfang Database, and Chinese Biomedical Literature Database of randomized controlled trials . beginning from their inception to August 2020. The primary outcomes is that Complex area of white spot and Percentage of Clinical Effectiveness will be accepted as the primary outcomes.Additional outcome is The safety assessment will be considered a secondary outcome. Two independent authors will based on the Cochrane system evaluation manual 5.1.0 version of RCT bias risk assessment tool to evaluate the risk of bias among the final included studies.And we will use the RevMan 5.3 software to analysis data. conclusion: This study will provide evidence to judge whether fire needle is an effective therapy for vitiligo.

\section{METHODS}

Participant or population: Studies have shown that vitiligo patients in the world have reached $1 \%$ of the total population, and the incidence rate in China is $0.553 \%$. Therefore, we can conclude that vitiligo patients in China have nearly about 8000000.

Intervention: The treatment group will receive fire needle therapy and can be combined with external application of drugs and narrow-band violet External line, 308 excimer light, traditional Chinese medicine and other methods, without any restrictions on needle material, shape, or treatment process.

Comparator: The control group will receive an internationally recognized therapy such as external application of drugs and narrow-band violet External line, 308 excimer light, Placebo, no treatment, but not use fire needle treatment.

Study designs to be included: All RCTs of fire needle therapy for vitiligo will be included without language restriction. NonRCTs, observational studies, cross-over studies, uncontrolled trials, animal trials, and reviews will be excluded.

Eligibility criteria: Inclusion criteria for study populations will be all patients with vitiligo. No restrictions will be applied in terms of gender, age, race, condition duration, or intensity. The language is limited to Chinese and English.

Information sources: The following electronic databases will be searched: PubMed, Embase, the Cochrane Library, the China National Knowledge Infrastructure, Chinese Science and Technology Periodical Database, Wanfang Database, and Chinese Biomedical Literature Database. We will search the databases from the beginning to AUGUST 2020.

Main outcome(s): Complex area of white spot and Percentage of Clinical 
Effectiveness will be accepted as the primary outcomes.

Additional outcome(s): The safety assessment will be considered a secondary outcome.

Quality assessment / Risk of bias analysis: Two independent authors will based on the Cochrane system evaluation manual 5.1.0 version of RCT bias risk assessment tool[6] to evaluate the risk of bias among the final included studies, the evaluation contents include: (1) randomized controlled trials; (2) whether allocation concealment is implemented; (3) whether the experiment is blind; (4) whether the evaluation of outcome indicators is blind; (5) whether the outcome indicators are complete; (6) whether selective reporting; (7) whether there are other bias risks. If there is ambiguity in the analysis, a third party will be added for negotiation.

Strategy of data synthesis: We will conduct statistical analysis through RevMan 5.3 software. For categorical data, we will calculate with the risk ratio (RR) and $95 \%$ confidence intervals (Cls). For continuous variables, mean difference (MD) will be included in the meta-analysis. If outcome variables are measured on different scales, results will be reported as standardized mean differences (SMDs) with $95 \% \mathrm{Cl}$.

Subgroup analysis: If the included studies have significant heterogeneity, we will perform subgroup analysis based on different control groups.

Sensibility analysis: When sufficient studies are available, sensitivity analysis will be used to assess the robustness of the metaanalysis based on methodological quality, sample size, and missing data.

Country(ies) involved: China.

Keywords: vitiligo; fire needle; meta analysis protocol.

Contributions of each author:

Author 1 - Liu Mingqiang.

Author 2 - Qiu Guirong. 